\title{
Single-Phase Pulse Width Modulated Rectifier
}

\author{
J. Bauer
}

The PWM rectifier is a very popular topic nowadays. With the expansion of electronics, conversion of electric parameters is also needed. For this purpose the side effects of passive rectifiers, e.g. production of harmonics and reactive power, must be taken into account. All these side effects fall away with the application of PWM rectifiers. This paper compares the differences between a phase angle controlled rectifier and a $P W M$ rectifier.

Keywords: Rectifier, Pulse Width Modulation, Harmonics Analysis, Active Front-End.

\section{Introduction}

At the beginning of the $19^{\text {th }}$ century, electric energy came into use in many technical fields. From the beginning ways were sought to change parameters such as voltage, frequency and current. The converters of electric parameters can be divided into two main groups. The first group uses for change the Faraday's law of induction e.g. the Ward-Leonard drive. The second group includes converters that work on the controlled switching principle, i.e., semiconducting rectifiers, inverters, etc.

\section{Phase controlled rectifiers}

The electric energy conversion made by semiconducting converters is being used more and more. This had led to the growth of negative phenomenons, that appeared negligible, when only a few converters being used. However the development of semiconductor structures has enabled higher power to be transmitted and has also led to wide spread of converters. In this way, converters have a negative effect on the supply network. The regressive effects of overloads with harmonics and reactive power consumption are becoming major disadvantages of phase controlled (mostly thyristor) rectifiers. These side effects need to be compensated by additional filtering circuits with capacitors or inductances. However, such circuits raise the costs and also increase material and space requirements for the converter.

Phase control and commutation of semiconducting devices impact the phase displacement between the first harmonics of the consumed current and the first harmonics of the supply voltage. This displacement leads to power factor degradation and to reactive power consumption. The consumed current harmonics cause non-sinusoidal voltage drops on the supply network impedances and lead to supply voltage deformation. This may cause malfunctions of other devices that are sensible to the sinusoidal shape of the supply voltage (e.g. measurement apparatuses, communication and control systems). The reactive power rises with longer control angle delays, so the rectifier acts as a time variable impedance that is nonlinear and causes deformed current consumption.

\section{$3 \mathrm{PWM}$ rectifiers}

In order to suppress these negative phenomena caused by the power rectifiers, use is made of rectifiers with a more sophisticated control algorithm. Such rectifiers are realized by semiconductors that can be switched off IGBT transistors. The rectifier is controlled by pulse width modulation. A rectifier controlled in this way consumes current of required shape, which is mostly sinusoidal. It works with a given phase displacement between the consumed current and the supply voltage. The power factor can also be controlled and there are minimal effects on the supply network.

PWM rectifiers can be divided into two groups according to power circuit connection - the current and the voltage type.

For proper function of current a type rectifier, the maximum value of the supply voltage must be higher than the value of the rectified voltage. The main advantage is that the rectified voltage is regulated from zero. They are suitable for work with DC loads (DC motors, current inverters)

For proper function voltage type rectifiers require higher voltage on the DC side than the maximum value of the supply voltage. The rectified voltage on the output is smoother than the output voltage of the current type rectifier. they also require a more powerful microprocessor for their control. Output voltage lower than the voltage on input side can be obtained only with increased reactive power consumption.

The function of the rectifier depends on the supply type of network. There are two types of supply network - "hard" and "soft".

Ordinary rectifiers, which work on a relatively "hard" supply network, do not affect the shape of the supply voltage waveform. Harmonics produce electromagnetic distortion, and the network will be loaded with reactive power. The PWM rectifier aims to consume sinusoidal current and to work with given power factor.

A PWM rectifier connected to the "soft" supply network has more potential to affect the shape of the supply voltage network. It can be controlled, so the current consumed by the PWM rectifier will partly compensate the non-harmonic consumption of other devices connected to the supply network.

\section{Control of the PWM rectifier}

The basic block diagram of one phase PWM rectifier is shown in Fig. 1.

The rectifier consists of 4 IGBT transistors, which form a full bridge, the input inductance and the capacitor at the output. It is controlled by pulse width modulation. Supply voltage $U_{s}$ and the voltage at the rectifier input $U_{r}$ are sinusoidal waveforms separated by the input inductance. The 


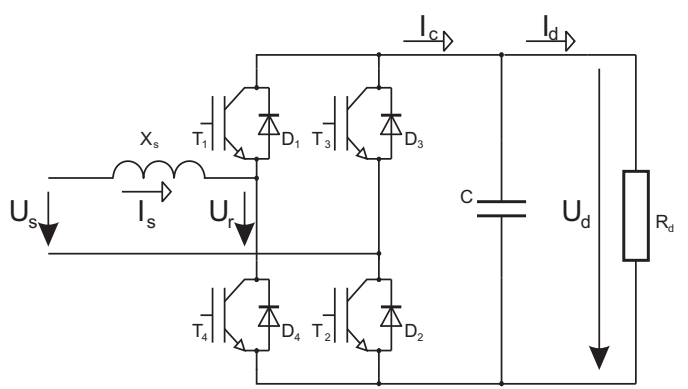

Fig. 1: Block diagram of the power section

energy flow therefore depends on the angle between these two phasors. See the phasor diagram in Fig. 2a.
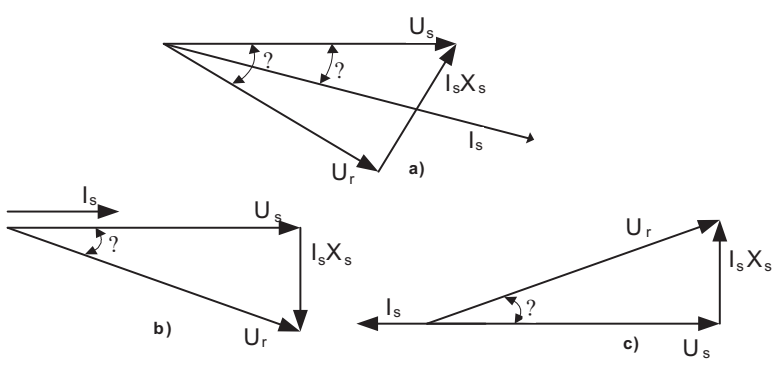

Fig. 2: Phasor diagrams

The power transferred from the supply to the input terminals of the rectifier is:

$$
\begin{aligned}
& P_{S R}=\frac{U_{s} U_{r}}{X_{s}} \sin \delta \\
& =U_{s} I_{s} \cos \phi,
\end{aligned}
$$

where $U_{s}$ RMS value of input supply voltage (V),

$U_{r}$ RMS value of first harmonics consumed by AC rectifier input $(\mathrm{V})$,

$\delta$ phase displacement between phasors $U_{s}$ a $U_{r}$,

$X_{s}$ input inductor reactance at $50 \mathrm{~Hz}(\Omega)$,

$\phi$ power factor.

In order to make the rectified voltage constant, the input and output powers must be balanced. Then, as the phasor diagram in Fig. 2a shows:

$$
\begin{aligned}
& I_{s} \cos \phi=U_{r} \frac{\sin \delta}{X_{s}}, \\
& I_{s} \sin \phi=\frac{U_{s}-U_{r} \cos \delta}{X_{s}} .
\end{aligned}
$$

As long as the reactive power consumed is equal to zero, the power factor is equal to unity. Therefore (3) and (4) can be adapted to:

$$
\begin{aligned}
& I_{s} X_{s}=U_{r} \sin \delta, \\
& U_{s}=U_{r} \cos \delta .
\end{aligned}
$$

Phasor diagrams of the rectifier, which works both, as a rectifier, and as an inverter, are shown in Figs. 2b and 2c.

The aim is to control the rectifier in such a way that it consumes harmonical current from the supply network, which is in phase with the supply voltage. This can be achieved by

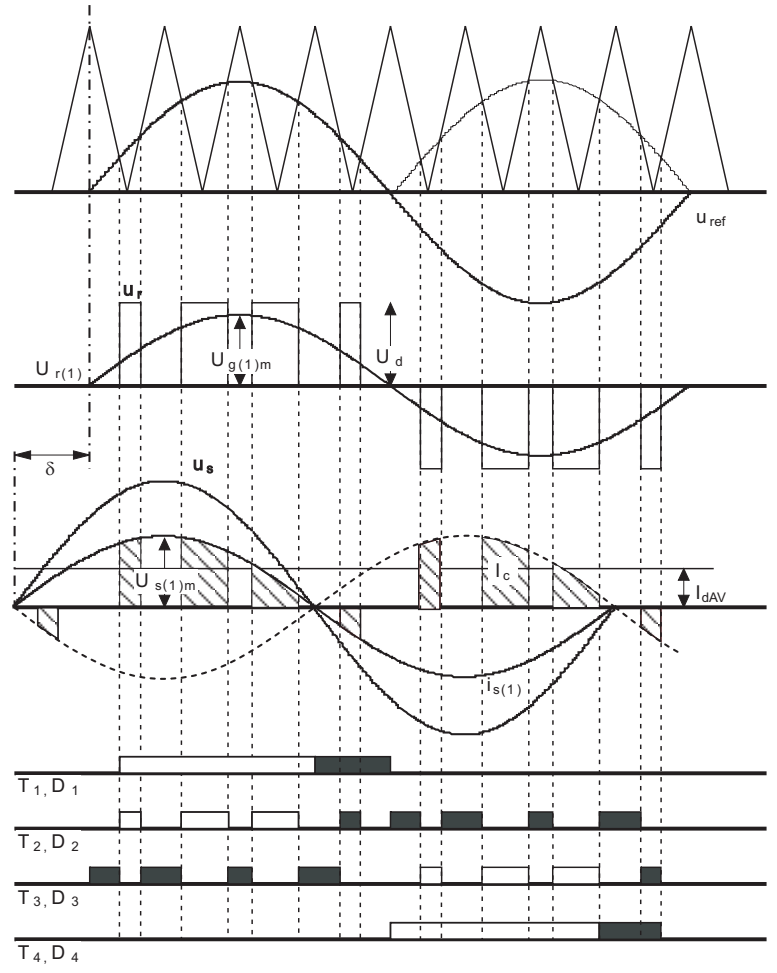

Fig. 3: Voltage and current waveforms in a PWM rectifier

controlling the rectifier in one of a number of ways, e.g., by pulse width modulation. Voltage and current under this control are shown in Fig. 3.

One possible way of transistor switching is shown at the bottom of Fig. 3. It can be seen that two states alternate. First, the current flows into the load ( $\mathrm{D}_{1}$ and $\mathrm{D}_{2}$ conducts), and second, the input of the rectifier is shortcircuited $\left(D_{1}\right.$ and $T_{3}$ conducts). The grey areas mean that the transistor conducts. The white areas mean, that the passive element conducts. The transistor is turned off and the current flows through the antiparallel diode. The switching of devices must be precisely synchronized with the supply voltage.

The output voltage of the rectifier $U_{d}$ is usually controlled to a constant value by using another converter e.g. an inverter. It is therefore possible, at a given current $I_{d}$ at the converter output, to assign to output voltage $U_{d}$ a particular value of $z$ and $\delta$. If we consider $\cos \varphi=1$ and the power equality on the AC and DC side can be written as:

$$
\begin{aligned}
& I_{d}=\frac{z \cdot I_{s(1) m}}{2} \cos \delta, \\
& I_{s(1) m}=\frac{2 U_{d A V} I_{d A V}}{U_{s m}} .
\end{aligned}
$$

From the phasor diagram in Fig. 2b

$$
\begin{aligned}
& \delta=\arctan \frac{\omega L I_{s(1) m}}{U_{s m}}, \\
& U_{g(1) m}=\frac{U_{s m}}{\cos \delta} .
\end{aligned}
$$

The rectifier controller can be made on the basis of these equations. It is obvious that only two variables are needed for such rectifier control. Firstly, the displacement angle $\delta$ must be controlled and the other necessary variable is $z$, 


$$
z=\frac{u_{r e f}}{u_{\text {pil }}},
$$

which is defined as the ratio of the modulation signal $u_{\text {ref }}$ amplitude to the saw-voltage $u_{p i l}$ amplitude. During displacement angle $\delta$ control, it is necessary to consider, that if $\delta$ increases the current through the inductance, will also increase.

For proper rectifier function, it is also necessary to synchronize the control algorithm with the supply voltage. By detecting the zero crossing, the controller controls the displacement angle $\delta$. The synchronization circuit must be very accurate and also fast and reliable.

\section{Measurement results}

A functional sample of the PWM rectifier was built. The control algorithm was realized on the basis mentioned above. Four IGBT transistors act as the power part, and a MOTOROLA 56F508 controller microprocessor was chosen. Simplified block diagram is shown in Fig. 4.

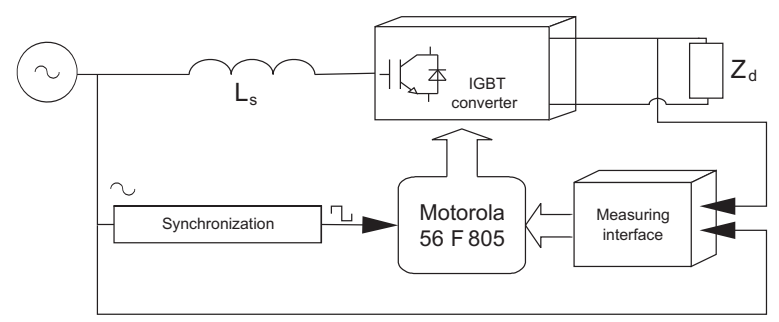

Fig. 4: Simplified block diagram of the PWM rectifier

An analog synchronization circuit was chosen, which later proved to be a bad idea. Zero cross detection did not function properly. The transistor switching disturbed the synchronization output.

The waveforms obtained from the measurements are shown in Figs. 5-8.

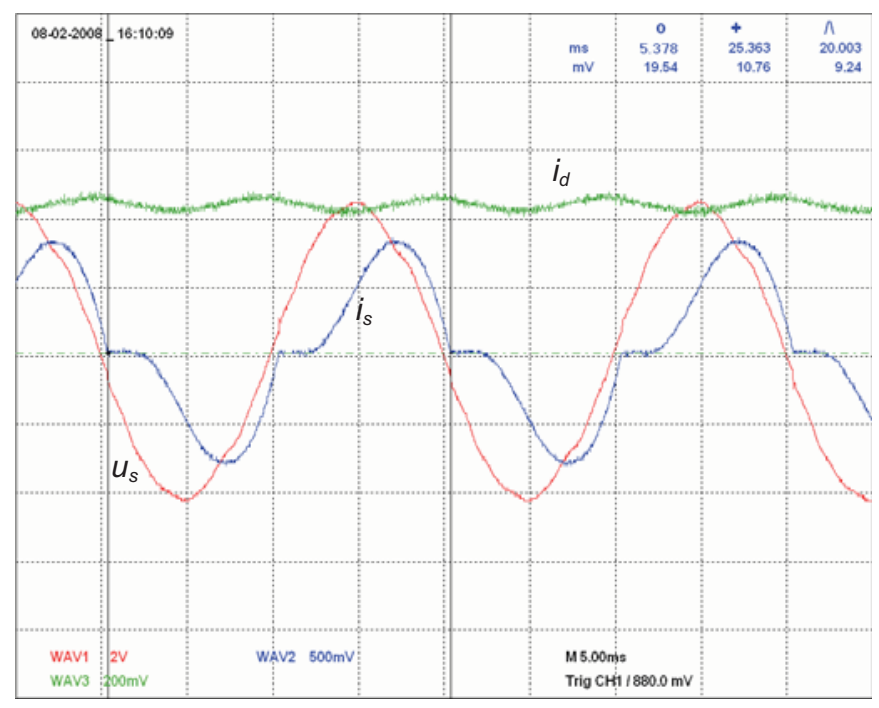

Fig. 5: Waveforms of the diode rectifier

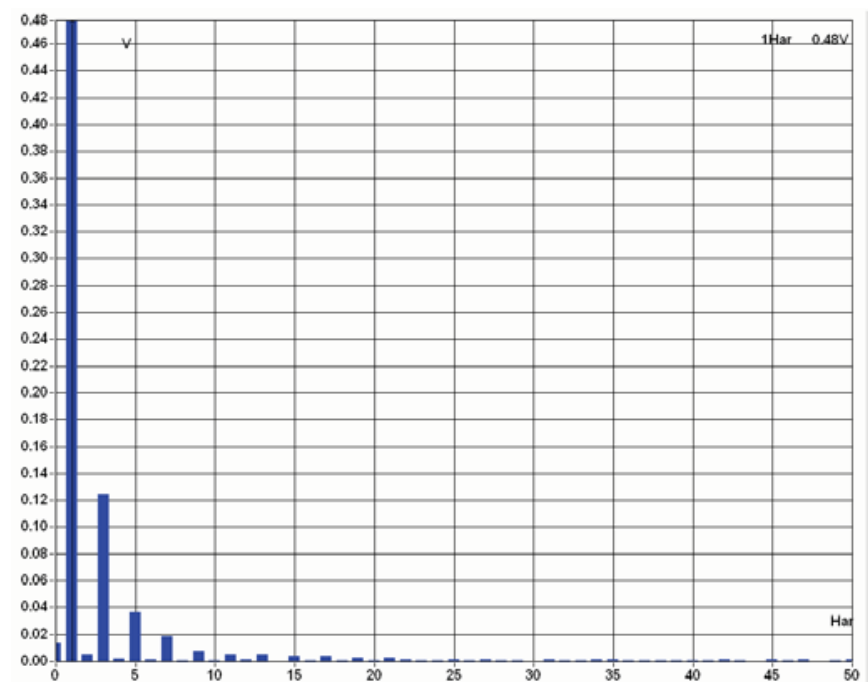

Fig. 6: Harmonics analysis of the diode rectifier input current

Fig. 5 shows typical diode rectifier waveforms. It can be seen that the current consumed from supply $i_{s}$ is non-sinusoidal.

A harmonics analysis of this current is shown in Fig. 6. The amplitudes of the third, fifth, etc., harmonics are high.

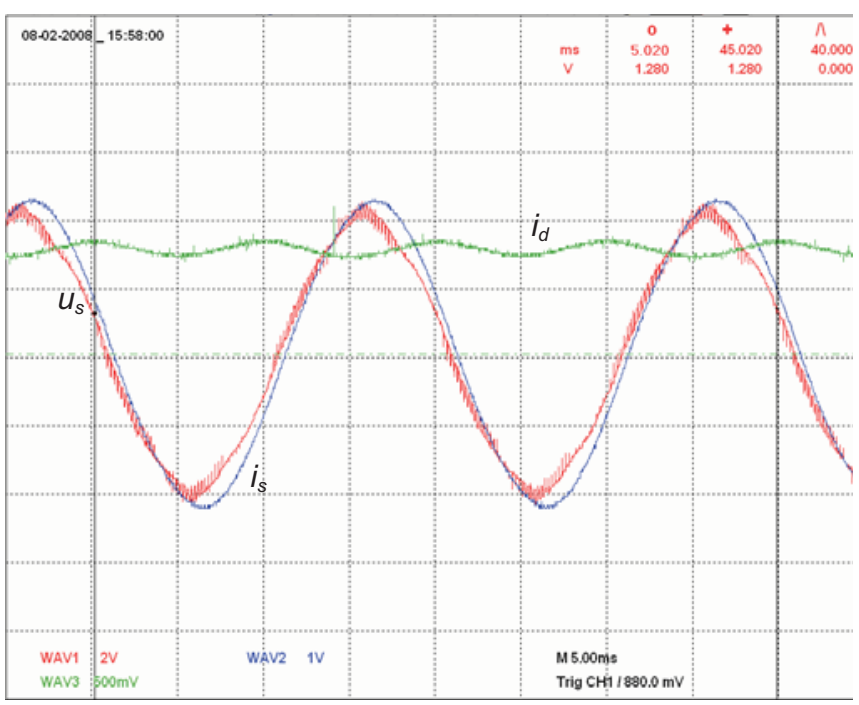

Fig. 7: Waveforms of the PWM rectifier

Fig. 7 shows the waveforms taken with PWM control of the rectifier. The current is is nearly sinusoidal and in phase with the supply voltage.

A harmonics analysis of the rectifier controlled by pulse width modulation is shown in Fig. 8.

The difference between these currents can be clearly seen.

The non-sinusoidal shape of the consumed current can be expressed by the harmonic coefficients.

$$
v=\frac{I_{(1)}}{I},
$$




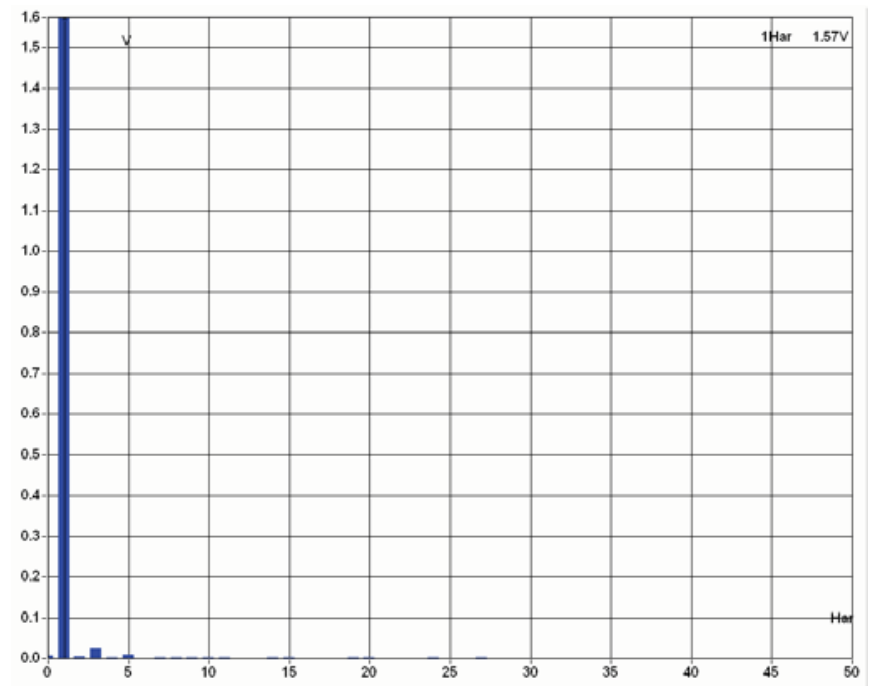

Fig. 8: Harmonics analysis of the PWM rectifier input current

$$
\begin{aligned}
& k_{h}=\frac{\sqrt{\sum_{2}^{\infty} I_{(n)}^{2}}}{I_{(1)}}, \\
& k_{z}=\frac{\sqrt{\sum_{2}^{\infty} I_{(n)}^{2}}}{I} .
\end{aligned}
$$

Then the coefficients calculated for the measurements are in Table 1.

Table 1 Harmonic coefficients

\begin{tabular}{|c|c|c|}
\hline Coefficient & Diode rectifier & PWM rectifier \\
\hline$v$ & $93 \%$ & $100 \%$ \\
\hline$k_{h}$ & $40 \%$ & $2 \%$ \\
\hline$k_{z}$ & $37 \%$ & $2 \%$ \\
\hline
\end{tabular}

\section{Conclusion}

The use of PWM control in rectifiers eliminates the problems caused by using phase controlled rectifiers. The PWM rectifier can perform well in many applications, for example as an active filter, or as an input rectifier for an indirect frequency converter. This application is useful mainly in traction, where the AC voltage from the trolley wire is first rectified, and the traction inverters and also other auxiliary converters are fed from the output of the rectifier. A traction vehicle equipped with a PWM rectifier does not consume reactive power, will not load the supply network with harmonics and can recuperate.

Another possible application of the converter is as an active filter. An active front-end will have the capacitor at the output.

\section{Acknowledgments}

The research described in the paper was supervised by Doc. J. Lettl CSc., FEE CTU in Prague.

\section{References}

[1] Lettl, J., Žáček, J.: Usměrňovače s šířkově pulzní modulací. Elektro, 1995.

[2] Künzel, K., Lettl, J., Žáček, J.: Some problems of PWM rectifiers. Proceedings of UEES 96, Szczecin, 1996.

[3] Lettl, J.: Duality of PWM rectifiers. Proceedings of Electronic Devices and Systems Conference 2003, Brno, 2003.

Ing. Jan Bauer

e-mail: bauerj1@.fel.cvut.cz

Department of Electric Drives and Traction

Czech Technical University in Prague

Faculty of Electrical Engineering

Technická 2

16627 Praha, Czech Republic 